\title{
Multimodal Video Summarization via Time-Aware Transformers
}

\author{
Xindi Shang* \\ shangxin@comp.nus.edu.sg \\ National University of Singapore \\ Singapore \\ Anran Wang \\ anran.wang@bytedance.com \\ ByteDance Inc. \\ Singapore
}

\author{
Zehuan Yuan $^{\dagger}$ \\ yuanzehuan@bytedance.com \\ ByteDance Inc. \\ China \\ Changhu Wang \\ wangchanghu@bytedance.com \\ ByteDance Inc. \\ China
}

\begin{abstract}
With the growing number of videos in video sharing platforms, how to facilitate the searching and browsing of the user-generated video has attracted intense attention by multimedia community. To help people efficiently search and browse relevant videos, summaries of videos become important. The prior works in multimodal video summarization mainly explore visual and ASR tokens as two separate sources and struggle to fuse the multimodal information for generating the summaries. However, the time information inside videos is commonly ignored. In this paper, we find that it is important to leverage the timestamps to accurately incorporate multimodal signals for the task. We propose a Time-Aware Multimodal Transformer (TAMT) with a novel short-term order-sensitive attention mechanism. The attention mechanism can attend the inputs differently based on time difference to explore the time information inherent inside video more thoroughly. As such, TAMT can fuse the different modalities better for summarizing the videos. Experiments show that our proposed approach is effective and achieves the state-of-the-art performances on both YouCookII and open-domain How2 datasets.
\end{abstract}

\section{CCS CONCEPTS}

- Computing methodologies $\rightarrow$ Video summarization.

\section{KEYWORDS}

video description, time-aware, multimodal summarization

\section{ACM Reference Format:}

Xindi Shang, Zehuan Yuan, Anran Wang, and Changhu Wang. 2021. Multimodal Video Summarization via Time-Aware Transformers. In Proceedings of the 29th ACM International Conference on Multimedia (MM '21), October 20-24, 2021, Virtual Event, China. ACM, New York, NY, USA, 10 pages. https://doi.org/10.1145/3474085.3475321

\footnotetext{
${ }^{*}$ Work done during internship at ByteDance AI Lab.

${ }^{\dagger}$ Corresponding author.
}

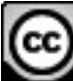

\section{(i)}

This work is licensed under a Creative Commons Attribution International 4.0 License MM '21, October 20-24, 2021, Virtual Event, China. (C) 2021 Copyright held by the owner/author(s). ACM ISBN 978-1-4503-8651-7/21/10.

https://doi.org/10.1145/3474085.3475321

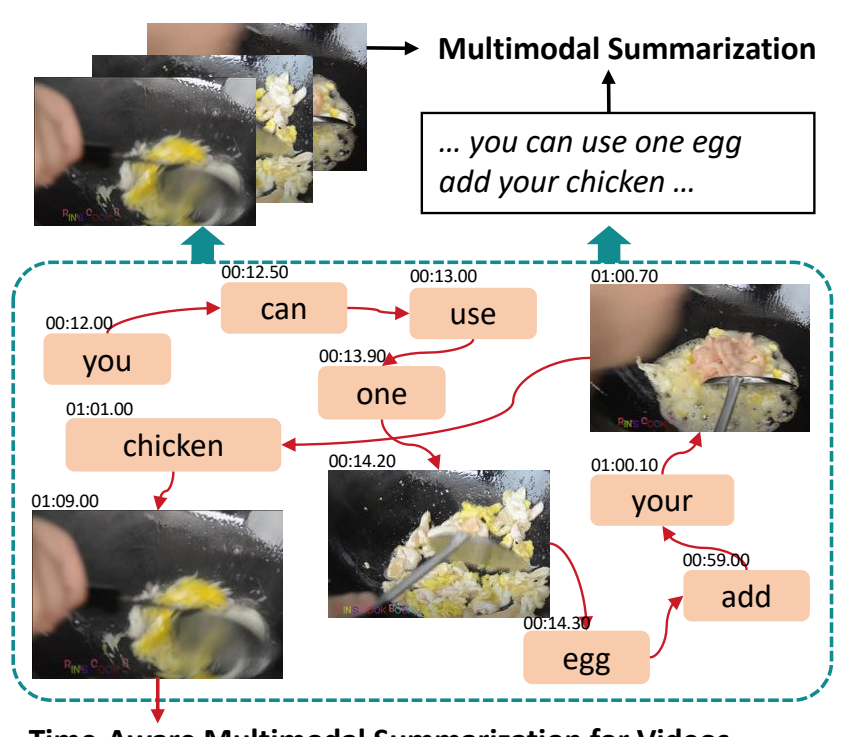

Time-Aware Multimodal Summarization for Videos

Figure 1: Illustration of multimodal video summarization. As can be seen, time information offers important clues to better fuse the vision and speech modalities for the task.

\section{INTRODUCTION}

Videos tend to comprise multiple modalities these days. In fact, the holistic presentation of instructional videos, movie commentaries and vlogs etc., which are prevalent on Internet nowadays, rely on not only the visual elements but also narrations. The narrations often convey the purposes or sentiments of video creators in addition to the mention of some visual elements. This suggests that the equation video $\approx$ vision may no longer hold for the videos of these types; and incorporating multimodal analysis becomes essential to fully understand their content and automatically generate short descriptions for summary. In this paper, we term this technique multimodal video summarization. The generated description would provide users with a quick overview of the content and assist search engines to efficiently retrieve the video, which are vital to video sharing platforms.

Different from video captioning which focuses on detailed descriptions for video clips [1], multimodal video summarization aims to generate summarized descriptions for longer videos [34]. On the one hand, this requires the model to additionally recognize the 


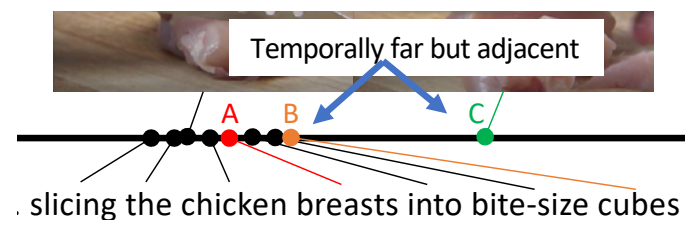

Figure 2: Illustration of temporally far but adjacent signals (B and $C$ ) in video. As comparison, $A$ and $B$ are not adjacent but temporally closer.

salient content of the video. On the other hand, multimodal video summarization challenges the model to both understand and consolidate the information from different modalities. These modalities may provide complementary information which is helpful to recognize some important content in the video. For example, it is hard to distinguish different kinds of meat simply based on the second frame in Figure 1, while the mention of "chicken" in the narration directly indicates the type of meat used in this cooking video. However, the multiple modalities inevitably introduce more redundancy and noise, such as the redundant frames and irrelevant content in the narration. These bring even more challenges in recognizing the salient content of the whole video and utilizing the complementarity for precise summaries.

To achieve the goal, the approaches for the general multimodal summarization can be directly adopted (the top of Figure 1). These approaches take a pair of text and image/video as the input, and generate the summary mainly through the encoder-decoder models. For instance, Chen and Zhuge [6] proposed a hierarchical attention model that uses two RNN encoders to obtain the respective text and image representations and a RNN decoder with multimodal attention to generate the summary. Based on a similar approach, Palaskar et al. [34] tackles the multimodal video summarization by treating the narration as the paired text of the video. Specifically, the text is obtained through automatic speech recognition (ASR) over the audio channel of the video. However, such approach will struggle to alleviate the interference from long-term redundancy and suppress the noises during multimodal fusion. Liu et al. [25] thus proposed a dedicated fusion forget gate to filter some noisy cross-modal alignments based on low-level features.

In this paper, we propose to utilize the timestamps that are inherently associated with the visual frames and ASR tokens, to overcome the challenges in multimodal video summarization. As can be seen in Figure 1, our approach treats the narration as the enclosed dynamic text instead of the surrounding static text like the previous works. This will explore the important time axis of the video more thoroughly. The direct benefits of such approach are two folds. (1) Given the time information, we can leverage the temporal distance to easily identify the long-term redundancy for both the visual signals ${ }^{1}$ and speech signals. This would be particularly helpful to separate those temporally far but adjacent signals (Figure 2) which are likely to be irrelevant or redundant, and alleviate their interference. (2) Along the same time axis, the visual and speech signals can be seamlessly organized in an unified manner, and thus the temporal relevance can provide extra useful evidences to determine whether the cross-modal signals are probably related. This would allow the model to concentrate within a time window

\footnotetext{
${ }^{1}$ Visual frames are usually sampled at different frequency before fed into the model.
}

during the multimodal fusion, such that being more robust to the noises in inaccurate cross-modal alignments.

To materialize our idea, we propose a Time-Aware Multimodal Transformer (TAMT) model. As illustrated in Figure 4, the model is based on the framework of recent Multimodal Transformer (MT) as in [15]. In order to leverage the time information inherent in videos, TAMT is empowered by a novel Short-Term Order-Sensitive Attention (STOSA) mechanism. While attending to the multimodal signals, TAMT separates them by short-term and long-term based on the temporal distance. In the short-term, TAMT is sensitive to the full order of the signals to capture the fine-grained sequential pattern for multimodal fusion. In the long-term, TAMT only considers the partial order of before/after, such that the signals that are temporally far away are treated as the contextual information. It is worth noting that such design is quite different from existing MTs which are only aware of the positional offset. As validated in the experiments, our proposed TAMT can generate more precise and diverse summaries compared to the previous works that neglect the time axis in the multimodal video summarization.

In summary, the contributions of this work include: (1) we propose to leverage the inherent time information inside video for better multimodal video summarization, typically by organizing the visual frames and ASR tokens in the unified time axis; (2) we propose an effective time-aware multimodal transformer (TAMT) with novel short-term order-sensitive attention mechanism to generate summaries from the inputs; and (3) we achieve the state-of-the-art performances on two challenging video datasets, the YouCookII [63] and open-domain How2 [40] datasets.

\section{RELATED WORK}

\subsection{Multimodal Summarization}

Traditionally, multimodal summarization aims to compress or describe multimodal documents using a short text summary. Prior works have mainly explored text+image $[3,4,52]$ and video+audio+ text $[10,23]$ using extractive approaches, while some works also focused on the abstractive approaches [22, 65]. Recently, Sanabria et al. [40] introduced multimodal summarization for videos, where the enclosed narrations and visual frames form the multimodal input and output a summarized description for the video. Palaskar et al. [34] proposed the first model to encode each input modality independently using separate RNNs and then fuse the modalities using a hierarchical cross-attention mechanism during the decoding of summaries. Liu et al. [25] proposed multistage fusion with forget gate to tackle the noises resulted from the cross-modal alignment during the multimodal fusion. Unfortunately, these works still treat the narration and visual frames in the same way as those traditional approaches in this area, but ignore the inherent time information inside videos.

\subsection{Video Summarization}

The video summarization task refers to selecting a compact yet informative set of video frames for the given video. This task can be viewed as the visual version of extractive text summarization [33]. Since the objective is very different from ours, we do not review these works in details. Several typical works in this area include $[11,19,28,32]$. 


\subsection{Video Captioning}

Standard video captioning focuses on generating descriptions for video clips $[35,51,54,57]$. For longer videos, dense video captioning is proposed to first temporally localize salient clips in the video and then generate the descriptions for each of them respectively [21, 42]. Multi-perspective video captioning is proposed to describe the video from different object perspective [5]. Instead, our work focuses on generating a single summarized description for the long video, which has different technical challenges from theirs.

Besides, most works in video captioning mainly explore the visual modality only. Several prior works have explored the combination of visual and audio features [8, 12, 16, 38, 42, 55], but have reported only minor performance improvements. On the contrary, recent works have incorporated the output of ASR over the audio channel and reported significant improvement for the narrated videos. In particular, Shi et al. [44] proposed a dense video captioning model that independently encodes the ASR tokens into a feature vector and concatenates it with each of the frame features to obtain the fused cross-modal features. These features are then fed into an encoder-decoder based on LSTMs to simultaneously predict video segments of interest and generate summaries. Hessel et al. [15] proposed to use a full-attention based model (i.e. Multimodal Transformers) to explore the interdependency among the different modalities. Nonetheless, these works did not address the challenges of the redundancy and noise in the multiple modalities, thus may fail to generate precise description for summary purpose.

\subsection{Language-Visual Fusion}

The fusion of speech and vision has attracted much research attention recently. It is different from audio-visual fusion $[20,48,53]$ as the linguistic property of the speech signals makes it distinctive from the low-level audio signals. Most of the works have adopted various Transformers [49] and explored vision and language fusion in a self-supervised manner by learning the visual-linguistic representations from images and the associated descriptions [27, $46,61,62]$. In the video domain, works like VideoBERT [30, 31, 47] studied the similar problem by leveraging the large language corpus automatically generated from the speech in the instructional videos. Specifically, they pretrained Multimodal Transformers using BERT-like strategy [9], and improved various downstream tasks such as video captioning. Besides, Pasunuru and Bansal [37] and Ma et al. [29] specifically studied language-visual fusion on the task of live video commenting. Compare to our work, the fusion approaches explored in all of these reviewed works do not leverage the time information in videos also, because they either do not have access to this information in their tasks or simply ignore it. It is worth noting that Miech et al. [30] observed the importance of using the timestamps of speech tokens and video frames, but they only explored it in the training objective of their studied problem.

\subsection{Self-Attention Mechanism}

Self-attention has been widely studied and successfully applied in various tasks. Vaswani et al. [49] were the first to propose Transformer models which entirely rely on self-attention mechanism. Vanilla self-attention can be regarded as global attention because

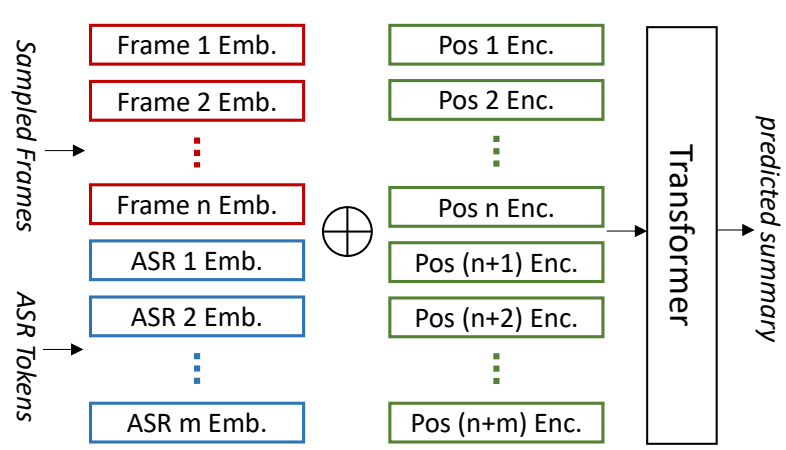

Figure 3: Illustration of positional encoding in the conventional Multimodal Transformer.

it attends to all the input elements. As extensions, some recent approaches explored local attention [7, 26, 45, 56, 59]. They restricted the attention to part of the input elements in order to handle longer input sequences. However, the global context is ignored in these approaches. To discriminate the global and local context, global-local attention is explored. Yang et al. [56] proposed to revise the original global attention map to be more focused on the local region using a learnable Gaussian bias. Zareian et al. [60] proposed to concatenate global and local attention heads for scene graph generation. Shaw et al. [41] proposed a relation-aware self-attention by treating the different distant relative positions as different embeddings. The different embeddings are used to represent the difference between the sequentially far and near elements in the input sequence. Inspired by this idea, several works have applied the relative position representation to video summarization [18] and modelling hierarchical structure in NLP tasks [39]. Similarly, our TAMT extends the idea to consider the time difference in the novel STOSA mechanism, such that it can better tackle the challenges in multimodal abstractive summarization.

\section{PROBLEM FORMULATION}

In this work, we particularly focus on using the video frames and textual transcripts generated by ASR as the input for multimodal video summarization. However, the proposed model can be generalized to more types of input modalities, e.g. audio feature. Let $X^{v}=\left(x_{1}^{v}, x_{2}^{v}, \ldots, x_{n}^{v}\right), x_{i}^{v} \in \mathbb{R}^{d_{\text {model }}}$ denote the sequence of visual embeddings, $X^{t}=\left(x_{1}^{t}, x_{2}^{t}, \ldots, x_{m}^{t}\right), x_{i}^{t} \in \mathbb{R}^{d_{\text {model }}}$ denote the sequence of ASR embeddings, and $X=\left\{x_{1}, x_{2}, \ldots, x_{n}\right\}$ alternatively denote the different modality embeddings when it is not necessary to distinguish the modality of $x_{i}$. Our target is to generate a summary consisting of a sequence of word tokens $Y=\left(y_{1}, y_{2}, \ldots, y_{L}\right)$ where $L$ is the maximum length of the summary.

For the existing approach such as Multimodal Transformer (MT), positional encodings are normally incorporated into the respective sequence $X$ in the encoder. Specifically, each element in $X$ is added a specific vector according to its position in the sequence, as illustrated in Figure 3. Apparently, such method only encodes the temporal order within each modality but not between modalities. The order of a video frame $i$ could be far away from that of an ASR token $j$ in the sequence even though they are temporally close to each other. As a result, models can only rely on the cross-modal 


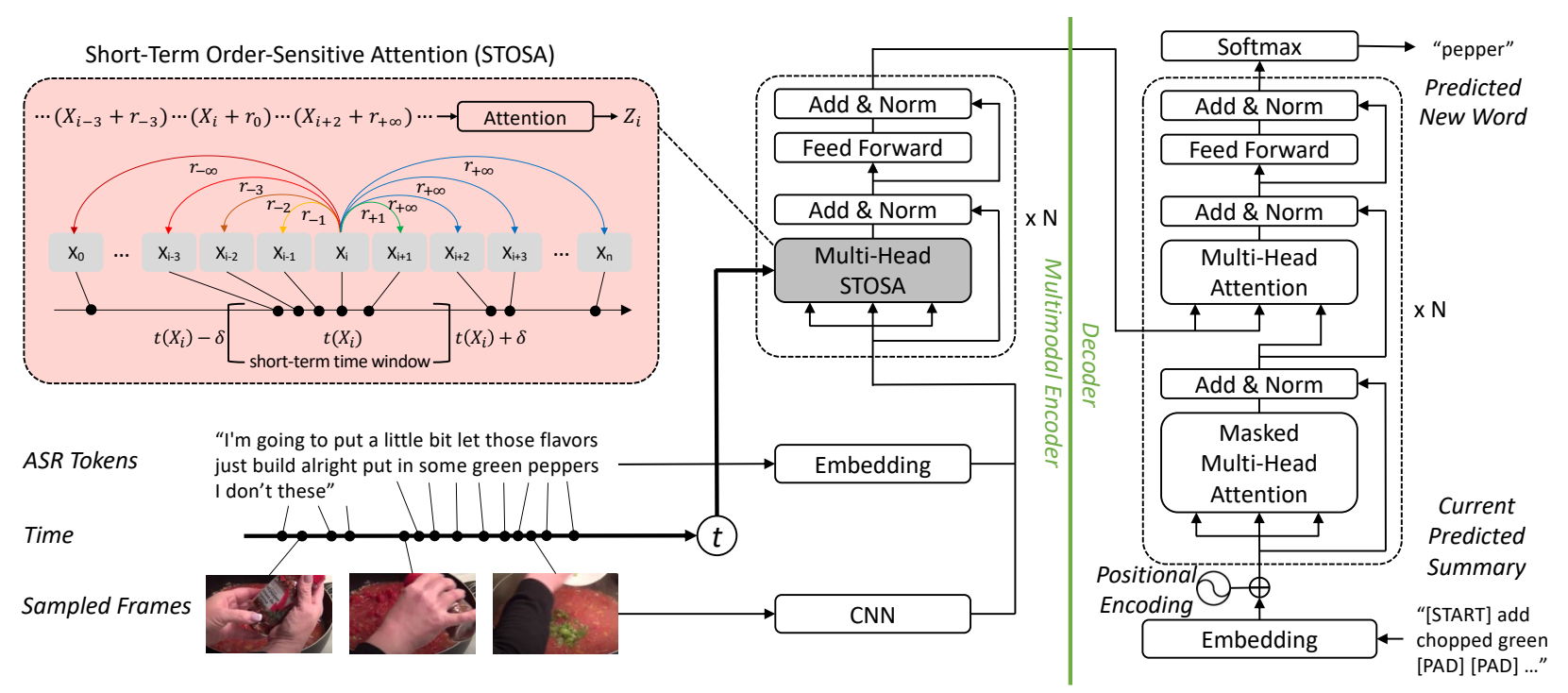

Figure 4: The framework of TAMT. The multimodal inputs of video frames and ASR tokens, together with the timestamps, are first fed into the multimodal encoder. The encoder consists of $\mathrm{N}$ blocks of short-term order-sensitive attention (STOSA). STOSA can distinctively model the short-term and long-term temporal relations between the elements and attend each element based on that. Then, a decoder consisting of $\mathrm{N}$ blocks of vanilla self-attention attends to the predicted tokens and encoder's outputs for generating a new word.

alignment based on the low-level features for the multimodal fusion. This will affect the robustness of the model in exploring the complementary effect between the visual and speech modalities, resulting in inaccurate summaries.

To address this issue, we believe that it is crucial to exploiting the associated timestamps of the video frames and ASR tokens. The inherent timestamps $t(x)$ can organize the input signals under a unified temporal order. This allows the model to know whether a sampled video frame and a ASR token is temporally close or far. Being aware of such cross-modal temporal relevance, the model should be more robust to noises in the cross-modal alignment while summarizing the video content. Additionally, the timestamps also allow the model to treat the signals in long-term differently to better alleviate their interference. As validated by our experiments, such an idea is effective to generate better summaries.

\section{THE TIME-AWARE MULTIMODAL TRANSFORMER}

As shown in Figure 4, our proposed TAMT consists of a multimodal encoder to encode the video input from multiple modalities and a decoder to generate the summaries. The model first passes the sampled video frames through convolutional networks (CNNs) to extract the visual features, and embeds them into the same space as the embeddings of ASR tokens. Then, $X^{v}$ and $X^{t}$ are concatenated into one sequence $X=\left\{x_{1}^{v}, \ldots, x_{n}^{v}, x_{1}^{t}, \ldots, x_{m}^{t}\right\}$, and fed into a stack of $N$ self-attention blocks. Meanwhile, as shown in the bottom-left of the figure, the inherent timestamps $t(x)$ organizes the input signals under a unified temporal order. Based on this, the encoder of TAMT uses a dedicated self-attention mechanism (namely STOSA) to consider the temporal relevance, and $t(x)$ are fed into each STOSA layer as illustrated by the bold arrow. To a certain extent, STOSA resembles the mechanism proposed in [41] which considers the relative position in the normal sequential order. However, the major difference is that the relative position between two elements $x_{i}, x_{j} \in X$ in the unified temporal order will change for different $X$, while that of [41] is always fixed at $i-j$.

\subsection{Short-Term Order-Sensitive Attention}

In fact, our short-term order-sensitive attention (STOSA) is sensitive to not only the unified temporal order but also the temporal distance. Despite the unified temporal order able to better reflect the temporal relevance between the visual and speech signals, some adjacent signals regarding this order could still be temporally irrelevant. This is due to the inconsistent frequency of the signals, such that some of the adjacent signals can be temporally very far. As a time-aware extension to the vanilla self-attention, STOSA particularly takes the timestamps into consideration with the intuition that shortterm temporal relations should be given more emphasis than the long-term temporal relations.

As illustrated in the top-left of Figure 4, STOSA denotes the input elements surrounding $x_{i}$ within a short time window as $S_{i}=$ $\left\{x_{j}|| t\left(x_{j}\right)-t\left(x_{i}\right) \mid<\delta, j=1,2, \ldots, n\right\}$, where $\delta$ denotes the time interval we consider. When performing self-attention for $x_{i}$, STOSA attends to $S_{i}$ and those elements outside the time window $X \backslash S_{i}$ with different sensitivity. Specifically, the compatibility between any of two elements in $X$ is computed as:

$$
e_{i j}= \begin{cases}\frac{x_{i} W^{Q}\left(x_{j} W^{K}+R_{i j}^{K}\right)^{T}}{\sqrt{d_{k}}}, & j \in S_{i} \\ \frac{x_{i} W^{Q}\left(x_{j} W^{K}+r_{-\infty}^{K}\right)^{T}}{\sqrt{d_{k}}}, & j \notin S_{i} \text { and } t(j)<t(i), \\ \frac{x_{i} W^{Q}\left(x_{j} W^{K}+r_{+\infty}^{K}\right)^{T}}{\sqrt{d_{k}}}, & j \notin S_{i} \text { and } t(j)>t(i)\end{cases}
$$


where $R_{i j}^{K} \in \mathbb{R}^{d_{k}}$ and $r_{-\infty}^{K}, r_{+\infty}^{K} \in \mathbb{R}^{d_{k}}$ are learnable vectors to represent the short-term and long-term temporal relations, respectively. $W^{Q} \in \mathbb{R}^{d_{\text {model }} \times d_{k}}, W^{K} \in \mathbb{R}^{d_{\text {model }} \times d_{k}}, W^{V} \in \mathbb{R}^{d_{\text {model }} \times d_{v}}$ are learnable parameter matrices.

As can be seen, distinctive representations $R_{i j}^{K}$ are used for different $x_{j}$ in the time window (w.r.t. $x_{i}$ ) to encode the short-term temporal order; whereas only two representations $r_{-\infty}^{K}, r_{+\infty}^{K}$ are used for the long-term temporal relation modeling to distinguish whether $x_{j}$ precedes or succeeds $x_{i}$. We define the short-term temporal relations as:

$$
R_{i j}^{K}=r_{\pi\left(x_{j}\right)-\pi\left(x_{i}\right)}^{K},
$$

where $\pi\left(x_{j}\right)$ is the position of $x_{j}$ in the ordered sequence of $S_{i}$, and $r^{K} \in \mathbb{R}^{M \times d_{k}} . M$ is the number of vectors in $r^{K}$ indicating different short-term relations within the time window. To determine its value, we estimate the maximum positional distance of the element pairs with time interval of less than $\delta$ in the training data:

$$
M=\max _{\left|t\left(x_{i}\right)-t\left(x_{j}\right)\right|<\delta}\left|\pi\left(x_{i}\right)-\pi\left(x_{j}\right)\right| \times 2 .
$$

Based on Eq.(1), STOSA then computes each output element $z_{i} \in \mathbb{R}^{d_{v}}$ as a weighted sum of transformed input elements:

$$
z_{i}=\sum_{j=1}^{n} \alpha_{i j}\left(x_{j} W^{V}\right), \alpha_{i j}=\frac{\exp e_{i j}}{\sum_{k=1}^{n} \exp e_{i k}} .
$$

In some cases, information about the temporal relations used by a given attention head is useful for subsequent layers. To propagate such information, we compute the output elements based on the temporal relation accordingly:

$$
\begin{aligned}
z_{i} & \left.=\sum_{j \in S_{i}} \alpha_{i j}\left(x_{j} W^{V}+R_{i j}^{V}\right)\right) \\
& +\sum_{j \notin S_{i}, t(j)<t(i)} \alpha_{i j}\left(x_{j} W^{V}+r_{-\infty}^{V}\right) \\
& +\sum_{j \notin S_{i}, t(j)>t(i)} \alpha_{i j}\left(x_{j} W^{V}+r_{+\infty}^{V}\right),
\end{aligned}
$$

where $R_{i j}^{V}=r_{\pi\left(x_{j}\right)-\pi\left(x_{i}\right)}^{V} \in \mathbb{R}^{d_{v}}$ and $\pi\left(x_{j}\right)$ is the same as that in Eq.(2). In addition, multi-heads are adopted in STOSA by simultaneously using $h$ different attention heads with the setting $d_{k}=d_{v}=d_{\text {model }} / h$. Unlike the $W^{Q}, W^{K}, W^{V}$ that are different across different heads, we share the $R_{i j}^{K}, R_{i j}^{V}, r_{-\infty}^{K}, r_{-\infty}^{V}, r_{+\infty}^{K}, r_{+\infty}^{V}$ among the heads in each layer. Finally, the $z_{i}$ output by different heads are concatenated and passed through a feed forward network, and then output to the next block.

Complexity Analysis. Suppose that $b$ is the batch size, $h$ is the number of attention heads, and $n$ is the length of the input sequence. As different attention heads sharing the same weights, the complexity of storing the extra representations for the short-term and long-term temporal relations is $O\left(b n^{2} d_{\text {model }}\right)$. To compute the attention, the vanilla attention mechanism achieves efficiency through $b h$ parallel multiplications of $n \times d_{\text {model }}$ and $d_{\text {model }} \times n$ matrices. In our STOSA mechanism, this parallel multiplication is infeasible because the matrix multiplication is different for each input sequence due to the different temporal relations. However, we can achieve the similar parallelism for computing $e_{i j}$ using $b n$ parallel multiplications of $h \times d_{\text {model }}$ and $d_{\text {model }} \times n$ matrices, by applying certain splitting and transpose operations to Eq.(1).

\subsection{Decoder for Summary Generation}

The decoder of TAMT follows the general scheme in Transformers. For each output token, the decoder attends to the previously generated summary through a masked attention, as well as the cross-modal representations extracted by the encoder from $X^{v}$ and $X^{t}$. To keep track of the order of the word tokens, positional encodings are added to the embeddings of the summaries before the first layer of the decoder. We adopt the same positional encoding as that of [49] based on sinusoids of varying frequency. By repeatedly output new tokens, TAMT finally generates the summary as a sequence of word tokens $Y=\left(y_{1}, y_{2}, \ldots, y_{L}\right)$.

\section{EXPERIMENT}

\subsection{Datasets}

We conduct experiments on two video datasets that contain narrations in the videos, YouCookII [63] and open-domain How2 [40].

YouCookII is the largest human-annotated cooking video dataset, containing 2,000 YouTube cooking videos of 89 different recipes. The dataset has imperative summaries, e.g., "add tofu cubes and mix well”, annotated on video segments, resulting in 7.7 segmentlevel summaries per video and 8.8 words per summary on average. Following [15], we conduct all the experiments on a subset of YouCookII that have both of the YouTube video and summary annotation publicly available at the time of analysis (Aug 2020), and perform 10 -fold cross-validation with randomly sampled 80/10/10 train/dev/test splits (at video level). Specifically, 12,961 annotated segments from 1,675 obtained videos and with an average duration of $20 \mathrm{~s}$ are used, and the same splits are used for all the compared approaches.

How2 contains YouTube videos from a wide range of instructional topics such as yoga, make-up and sport, which are first collected by [58]. Unlike the dedicated annotators in YouCookII, the ground truth summaries in How2 are provided by the upload users. Such summaries are of more free forms including summaries like "learn the sivananda yoga single right leg raise in this free exercise video", and longer length of 33 words on average. Specifically, the dataset contains 73,993 videos for training, 2,965 for validation and 2,156 for testing, with an average duration of 90s. Since some videos has been removed from YouTube, we can obtain 68,237 videos from YouTube for training, 2,517 for validation and 2,125 for testing at the time of analysis.

\subsection{Implementation Details}

For YouCookII, we use the visual features released by [64]. The features include the appearance features output by ResNet-200 [14] and motion feature outputs by BN-Inception [17], extracted at every $0.5 \mathrm{~s}$ from the videos ${ }^{2}$. Following [15], we sample 10 frames for each YouCookII video segment, and feed the corresponding visual features into the model. For How2, we use the visual features officially released by [40], which are 2048-D feature vectors output

\footnotetext{
${ }^{2}$ We did not use the features provided by [63] because the timestamps of those features have been omitted in the release.
} 


\begin{tabular}{c|c|ccccccc}
\hline Dataset & Approach & BLEU-1 & BLEU-2 & BLEU-3 & BLEU-4 & METEOR & ROUGE-L & CIDEr \\
\hline \multirow{5}{*}{ YouCookII } & Visual Only [47] & - & - & 7.59 & 4.33 & 11.94 & 28.80 & 55.00 \\
& ASR Only [15] & - & - & - & 8.55 & 16.93 & 35.54 & 106.00 \\
& Shi's [44] & - & - & - & 2.76 & 18.08 & - & - \\
& MT [15] & 36.75 & 23.00 & 14.81 & 9.84 & 18.13 & 36.96 & 114.09 \\
& TAMT & $\mathbf{3 8 . 2 0}$ & $\mathbf{2 3 . 9 5}$ & $\mathbf{1 5 . 5 3}$ & $\mathbf{1 0 . 3 5}$ & $\mathbf{1 8 . 3 5}$ & $\mathbf{3 7 . 5 5}$ & $\mathbf{1 1 7 . 4 0}$ \\
\hline \multirow{5}{*}{ How2 } & Visual only [25] & 49.60 & 38.40 & 32.90 & 27.40 & 22.90 & 46.80 & 146.10 \\
& ASR only [25] & 49.80 & 38.40 & 32.00 & 27.60 & 22.80 & 45.80 & 155.10 \\
& Palaskar's [34] & 53.10 & 42.50 & 36.40 & 32.10 & 28.90 & 50.10 & 191.80 \\
& MT [15] & - & - & - & 37.78 & 31.52 & 54.23 & 254.32 \\
& Liu's[25] & 57.80 & 48.20 & 42.80 & 39.00 & 28.20 & 55.50 & 234.60 \\
& TAMT & $\mathbf{5 9 . 6 7}$ & $\mathbf{5 0 . 5 1}$ & $\mathbf{4 4 . 7 9}$ & $\mathbf{4 0 . 5 8}$ & $\mathbf{3 3 . 3 0}$ & $\mathbf{5 6 . 6 4}$ & $\mathbf{2 7 4 . 1 4}$ \\
\hline
\end{tabular}

Table 1: Performance comparison with the state-of-the-art approaches on YouCookII and How2. Shi's [44] and MT [15] are the multimodal approaches that incorporate the visual and ASR signals. Palaskar's [34] and MT [15] are the multimodal approaches that incorporate the visual and ASR signals.

by ResNeXt-101 pretrained to recognize 400 different actions [13] and extracted at every 16 frames from the videos. We sample 50 frames for each How2 video. All the frames are sampled evenly during testing, and randomly with replacement during training.

For both datasets, we use the transcripts automatically generated by YouTube's ASR engine ${ }^{3}$ and preserve the timestamp information of all the ASR tokens. To estimate the timestamp for each ASR token, we simply take the mean of the start and end timestamps output by the ASR engine for the token. The average length of transcript per video in YouCookII and How2 are 42 and 291, respectively. We truncate the transcript to the maximum length of 80 for YouCookII, and 600 for How2.

As for the model settings on YouCookII, we set $N=2, h=$ $8, d_{\text {model }}=256, L=15$, and the time interval $\delta$ in STOSA as 1.0s. For How2, we set $N=6, h=8, d_{\text {model }}=512, L=50$, and the time interval $\delta$ as $2.0 \mathrm{~s}$. We do not propagate the temporal relation information across layers for How2, as we found it is not useful and will result in overfitting. To train the models, we used a batch size of 128 for YouCookII, and 8 for How2. We use SGD optimizer with the starting learning rate of 0.1 , and reduce the learning rate by half whenever the ROUGE-L score on validation set does not increase for 1 epoch. We train the models for 50 epochs, and select the best model according to the ROUGE-L score on validation set.

\subsection{Comparison with the State-of-the-Arts}

Table 1 compares our proposed TAMT with the state-of-the-art approaches on YouCookII and How2 datasets. In particular, we compare the approaches using only single modality and the multimodal approaches $[15,34,44]$. We reimplemented Multimodal Transformer (MT) [15] under the above experimental settings (Section 5.2) on both YouCookII and How2 for a fair comparison. For $[25,34,44]$, we report the results from the previous works. We use various metrics including BLEU [36], METEOR [2], ROUGE-L [24] and CIDEr $[50]^{4}$ to evaluate the overall summarization performance.

\footnotetext{
${ }^{3}$ There are around 200 YouCookII videos with no auto-generated transcripts available on YouTube, so we obtain the transcripts for those videos through Google Cloud Speech-to-Text API.

${ }^{4}$ We adopt the implementations in MSCOCO evaluation toolkit: https://github.com/ tylin/coco-caption
}

\begin{tabular}{lcccc}
\hline & BLEU-4 & METEOR & ROUGE-L & CIDEr \\
\hline MT & 9.84 & 18.13 & 36.96 & 114.09 \\
MT+RP & 10.02 & 18.10 & 37.10 & 114.37 \\
\hline TAMT+RP & 9.99 & 18.14 & 37.29 & 115.56 \\
TAMT+STOSA & $\mathbf{1 0 . 3 5}$ & $\mathbf{1 8 . 3 5}$ & $\mathbf{3 7 . 5 5}$ & $\mathbf{1 1 7 . 4 0}$ \\
TAMT+LOCAL & 9.65 & 17.92 & 36.85 & 113.04 \\
\hline
\end{tabular}

Table 2: Ablation studies on model variants. RP stands for relative position encoding. LOCAL stands for local attention mechanism.

For the 10-fold cross-validation experiments on YouCookII, we report mean score over the 10 splits for each metric. For all the metrics, higher score indicates better performance.

Overall, we can see that the approaches that incorporate visual and ASR modalities perform significantly better. Particularly, the ASR Only approaches outperform the Visual Only. This demonstrates the effectiveness and importance of speech signals in the multimodal video summarization. Moreover, we can see that our TAMT outperforms all the compared approaches. In particular, the performances of TAMT are consistently superior to that of MT regarding all the metrics. We believe that such superiority is mainly due to the use of unified temporal order and STOSA in our model. Also, the performances of TAMT surpass MT by a larger margin on How2 than that on YouCookII. This suggests that TAMT is more effective when the videos or ASR token sequences are longer.

\subsection{Ablation Studies}

To further verify where the improvements are coming from in our proposed ideas, we conduct ablation studies on YouCookII dataset by comparing several model variants.

Effect of STOSA Mechanism. We implement MT and TAMT with the relative position (RP) encoding proposed in [41]. The difference between the two variants is that TAMT+RP models the relative position according to the unified temporal order, while MT+RP models the relative position simply according to the concatenation of video frame and ASR sequences. We set the maximum distance to 


\begin{tabular}{c|cc|cc}
\hline \multirow{2}{*}{$\delta$} & \multicolumn{2}{|c|}{ YouCookII } & \multicolumn{2}{c}{ How2 } \\
& ROUGE-L & CIDEr & ROUGE-L & CIDEr \\
\hline $0.5 \mathrm{~s}$ & $\underline{37.17}$ & $\underline{114.74}$ & 55.54 & $\underline{264.21}$ \\
$1.0 \mathrm{~s}$ & $\mathbf{3 7 . 5 5}$ & $\mathbf{1 1 7 . 4 0}$ & 55.73 & 264.59 \\
$1.5 \mathrm{~s}$ & 37.33 & 116.42 & 55.76 & 268.62 \\
$2.0 \mathrm{~s}$ & 37.23 & 115.13 & $\mathbf{5 6 . 6 4}$ & $\mathbf{2 7 4 . 1 4}$ \\
$2.5 \mathrm{~s}$ & 37.37 & 115.10 & 56.45 & 273.87 \\
$3.0 \mathrm{~s}$ & 37.41 & 115.55 & 56.40 & 272.78 \\
$3.5 \mathrm{~s}$ & 37.48 & 117.27 & 55.92 & 270.69 \\
$4.0 \mathrm{~s}$ & 37.34 & 116.23 & 56.02 & 273.05 \\
$4.5 \mathrm{~s}$ & 37.35 & 116.10 & 55.80 & 266.03 \\
$5.0 \mathrm{~s}$ & 37.30 & 114.82 & $\underline{55.44}$ & 267.66 \\
\hline
\end{tabular}

Table 3: The effect of different time interval $\delta$ on the model performance. The bold and underlined scores are the highest and lowest, respectively, for each column.

\begin{tabular}{c|c|ccc}
\hline & & Vocab Cov. & Novelty & Uniqueness \\
\hline \multirow{3}{*}{ YC2 } & MT & 48.88 & 75.65 & 78.81 \\
& TAMT & 48.66 & $\mathbf{7 8 . 8 9}$ & $\mathbf{8 2 . 8 8}$ \\
& GT & 76.11 & 92.71 & 98.00 \\
\hline \multirow{3}{*}{ How2 } & MT & 45.37 & 96.80 & 99.34 \\
& TAMT & 44.59 & 96.61 & 99.11 \\
& GT & 52.72 & 97.66 & 99.92 \\
\hline
\end{tabular}

Table 4: Diversity analysis of the summaries generated by TAMT and MT, compared to ground truth on YouCookII (YC2) and How2.

2 in the relative position encoding. This value is selected according to the best performance through grid search. From the top four rows in Table 2, we have the following observations. (1) TAMT+RP improves over MT more than the MT+RP does. This shows the superiority of unifying the temporal order of multimodal signals using their associated timestamps. Under the unified temporal order, the model is able to establish more accurate cross-modal correspondence. (2) TAMT+STOSA achieves the best performance among all the variants. The comparison with $\mathrm{TAMT}+\mathrm{RP}$ also verifies that our proposed STOSA can better utilize the time information than RP. In addition, our Student's t-test shows that these improvements from TAMT+STOSA over the compared variants are statistically significant with $\mathrm{p}$-value $<0.05$ for all the BELU, METOER and ROUGE-L metrics.

Besides, we also study the TAMT variant using the local attention mechanism. We implement it by restricting STOSA to only attend to the elements in the short time windows. The last row in Table 2 shows that such a variant will lead to significant performance drop compared to the baselines. Therefore, the global context (i.e. information outside the time window) is useful and cannot be ignored.

Effect of Time Window Size. As introduced in Section 4.1, the short-term time window size is controlled by the hyperparameter of time interval $\delta$, which is the special hyperparameter unique to the proposed STOSA. For $\delta=5 \mathrm{~s}$, the time window will include

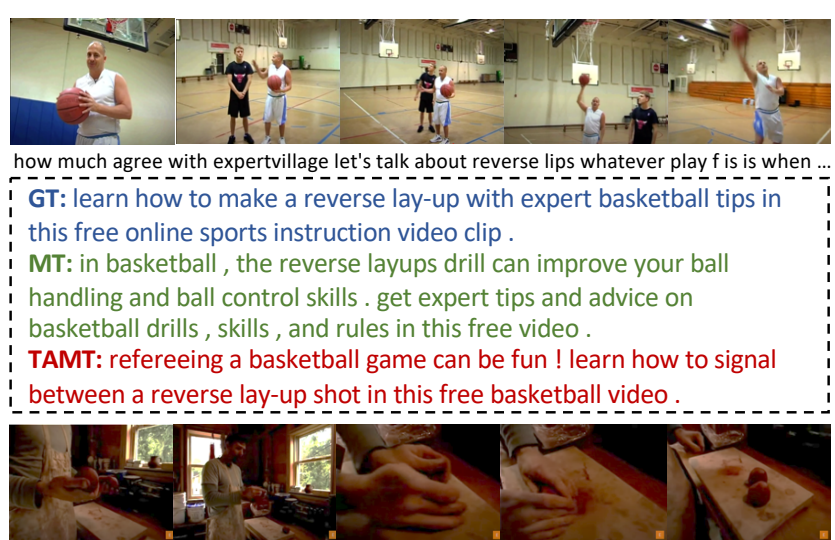

okay so we way. Clay and the next step in this process is now to wedge the clay the main ... GT: the main purpose of wedging clay for ceramic mugs is to extract air out of the clay. learn how to wedge clay to throw a mug from an artist in this free ceramics and pottery video .

MT: wedging clay for a ceramic mug creates a consistent clay body learn to wedge clay with tips from a potter in this free ceramics video. TAMT: wedging clay for a clay pot removes air bubbles and creates a consistent clay body . throw a clay mug by wedging air out the clay with tips from an artist in this free video on ceramics and pottery . - - - - - - - - - - - - - - - - - - - - - - - - - - - - - - - - - -

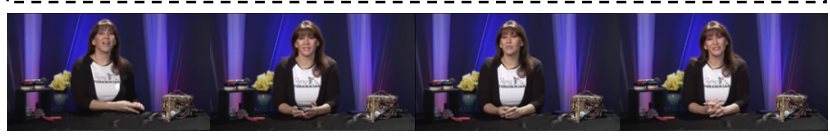

over productive sebaceous glands are oil glands are responsible for oily skin I'm Jackie ... GT: people with oily skin should wash their face two or three times a day with mild cleansers . fight oily skin with tips from a beauty editor in this free video on skin care.

MT: oily skin can be caused by over - productive <unk> glands, such as oil glands, <unk> glands and <unk> glands. fight oily skin with tips from a beauty editor in this free video on skin care

TAMT: fight oily skin by using a mild cleanser, a papaya glands, a mild cleanser and a mild cleanser. fight oily skin with tips from a beauty editor in this free video on skin care.

Figure 5: Qualitative examples generated by TAMT and MT on How2. As the transcript length (\# ASR tokens) is much longer, we only display the beginning tokens under each video due to the space limitation. Readers can refer to the GT to understand the real video content better.

around 22 ASR tokens on average, according to the average wordsper-minute (WPM) of speech in the datasets. To study its effect, we varies $\delta$ from $0.5 \mathrm{~s}$ to $5 \mathrm{~s}$, and report the corresponding performances of TAMT. Table 3 shows this performance variation. It worth noting that the minimum scores in this table still surpass the corresponding scores of the compared approaches in Table 1.

\subsection{Diversity of the Generated Summaries}

The above experiments analyse (Table 1) mainly the correctness of the generated summaries. To provide a more holistic view of the quality, we additionally analyse how diverse the generated summaries are. Following [15, 43], we measure the following corpuslevel diversity metrics. Vocab Coverage: the percent of vocabulary used in the generated summaries; Novelty: the percent of generated 

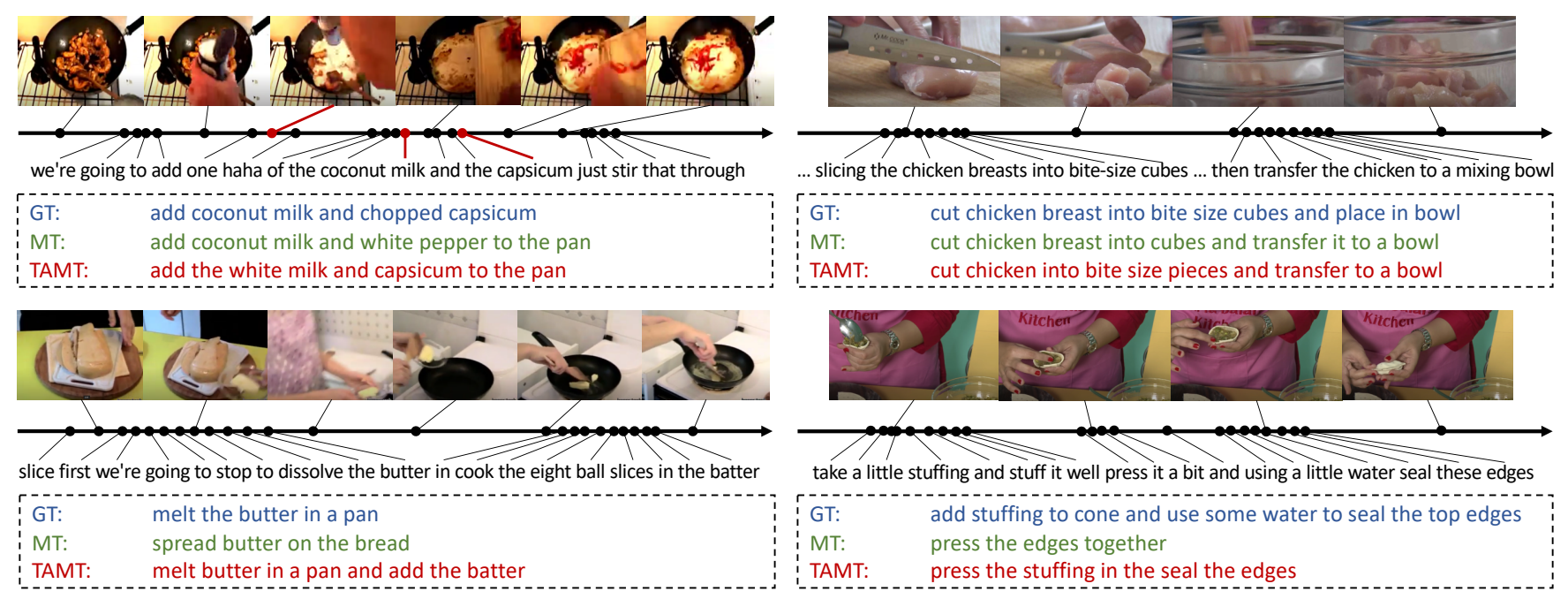

Figure 6: Qualitative examples generated by TAMT and MT on YouCookII. The timestamps of the video frames and ASR tokens is roughly reflected on the time arrows, for better visualizing the inconsistent frequency in the examples.

summaries not seen in the training set; and Uniqueness: the percent of generated summaries that are unique.

The top section of Table 4 shows that our TAMT generates more diverse summaries on YouCookII in terms of both novelty and uniqueness. We speculate that this is because TAMT can fuse more novel pairs of visual and ASR tokens by exploiting the temporal relevance, when the embeddings of the pairs cannot be tuned to be similar by the limited training data. By correlating to Table 1, we find that TAMT outperforms MT in both the correctness and diversity. Unlike YouCookII, the diversity of the generated summaries by TAMT is slightly lower than that of MT for How2. However, it is noteworthy that their diversity is both close to that of the ground truth. We conjure that this is because of the abundant training corpus available in How2 for the models to achieve such diversity. Therefore, despite the minor differences on diversity, we can still affirm the superiority of TAMT for its significantly higher correctness in Table 1.

\subsection{Qualitative Analysis}

Figure 6 displays several qualitative examples on YouCookII. Compare to the summaries generated by the state-of-the-art MT, we can see that the summaries generated by TAMT are generally more accurate and contain more details. Particularly, in the left-bottom example, TAMT is able to complement the summary with "in a pan" using the visual signal, even though the transcript does not mention the use of pan. In contrast, MT fails in this case. MT also makes a mistake in cross-modal correspondence, such as "white pepper" generated in the top-left example. As we can see from the red lines over the time arrow, such mistake is likely because MT is unaware of the lower temporal relevance between the visual "white" and ASR token "capsicum". The last example shows a failure case where TAMT fails to correctly describe some detailed action in the generated summary.
Figure 5 presents the qualitative examples on How2. As can be seen, the ground truth summaries of How2 contains more abstractive description and summary about the video content. This poses more challenges for the models to recognize the important information. However, for the presented examples, we can find that TAMT is able to summarize the videos with generally correct summaries about the intentions of the video creators. As comparison, MT either includes some unimportant details (e.g. "ball handling and ball control skill" for the 1st video about teaching reverse lay-up) or misses important information (e.g. "extract air out of clay" for the 2nd video). These mistakes could be imputed to the lack of unified temporal order for the model to recognize necessary important information based on the multimodal signals. The case will be harder if the video is mainly made by oral expression, as shown in the 3rd example. Summarizing this type of videos will also rely on the technique of natural language understanding. Hence, it is unsurprising to see that both TAMT and MT struggle to generate satisfactory summaries in the presented example.

\section{CONCLUSION}

In this paper, we propose to leverage the inherent time information inside video for better multimodal video summarization, which has been commonly ignored in the previous works. Therefore, we proposed a Time-Aware Multimodal Transformer (TAMT) for multimodal video summarization. In order to explore the time information thoroughly, we introduce a novel short-term order-sensitive attention mechanism in the encoder of our TAMT. Extensive experimental analyses on YouCookII and How2 validated the superiority of TAMT over the state-of-the-arts, and the effectiveness of our idea to utilizing the associated timestamps to organize the multimodal signals in videos. For future work, it is potential to explore TAMT in learning better visual-linguistic representations in a self-supervised manner. 


\section{REFERENCES}

[1] Nayyer Aafaq, Ajmal Mian, Wei Liu, Syed Zulqarnain Gilani, and Mubarak Shah 2019. Video description: A survey of methods, datasets, and evaluation metrics. ACM Computing Surveys (CSUR) 52, 6 (2019), 1-37.

[2] Satanjeev Banerjee and Alon Lavie. 2005. METEOR: An automatic metric for MT evaluation with improved correlation with human judgments. In Proceedings of the acl workshop on intrinsic and extrinsic evaluation measures for machine translation and/or summarization. 65-72.

[3] Jingwen Bian, Yang Yang, and Tat-Seng Chua. 2013. Multimedia summarization for trending topics in microblogs. In Proceedings of the 22nd ACM international Conference on information \& knowledge management. 1807-1812.

[4] Jingwen Bian, Yang Yang, Hanwang Zhang, and Tat-Seng Chua. 2014. Multimedia summarization for social events in microblog stream. IEEE Transactions on multimedia 17, 2 (2014), 216-228.

[5] Yi Bin, Xindi Shang, Bo Peng, Yujuan Ding, and Tat-Seng Chua. 2021. MultiPerspective Video Captioning. In Proceedings of the 29th ACM International Conference on Multimedia.

[6] Jingqiang Chen and Hai Zhuge. 2018. Abstractive text-image summarization using multi-modal attentional hierarchical rnn. In Proceedings of the 2018 Conference on Empirical Methods in Natural Language Processing. 4046-4056.

[7] Rewon Child, Scott Gray, Alec Radford, and Ilya Sutskever. 2019. Generating long sequences with sparse transformers. arXiv preprint arXiv:1904.10509 (2019).

[8] Shun-Po Chuang, Chia-Hung Wan, Pang-Chi Huang, Chi-Yu Yang, and Hung-Yi Lee. 2017. Seeing and hearing too: Audio representation for video captioning. In 2017 IEEE Automatic Speech Recognition and Understanding Workshop (ASRU). IEEE, 381-388.

[9] Jacob Devlin, Ming-Wei Chang, Kenton Lee, and Kristina Toutanova. 2019. BERT Pre-training of Deep Bidirectional Transformers for Language Understanding. In Proceedings of the 2019 Conference of the North American Chapter of the Association for Computational Linguistics: Human Language Technologies, Volume 1 (Long and Short Papers). 4171-4186.

[10] Georgios Evangelopoulos, Athanasia Zlatintsi, Alexandros Potamianos, Petros Maragos, Konstantinos Rapantzikos, Georgios Skoumas, and Yannis Avrithis. 2013. Multimodal saliency and fusion for movie summarization based on aural, visual, and textual attention. IEEE Transactions on Multimedia 15, 7 (2013), 15531568.

[11] Michael Gygli, Helmut Grabner, Hayko Riemenschneider, and Luc Van Gool. 2014. Creating summaries from user videos. In European conference on computer vision. Springer, 505-520.

[12] Wangli Hao, Zhaoxiang Zhang, He Guan, and Guibo Zhu. 2018. Integrating both visual and audio cues for enhanced video caption. In AAAI Conference on Artificial Intelligence.

[13] Kensho Hara, Hirokatsu Kataoka, and Yutaka Satoh. 2018. Can spatiotemporal $3 \mathrm{~d}$ cnns retrace the history of $2 \mathrm{~d}$ cnns and imagenet?. In Proceedings of the IEEE conference on Computer Vision and Pattern Recognition. 6546-6555.

[14] Kaiming He, Xiangyu Zhang, Shaoqing Ren, and Jian Sun. 2016. Deep residual learning for image recognition. In Proceedings of the IEEE conference on computer vision and pattern recognition. 770-778.

[15] Jack Hessel, Bo Pang, Zhenhai Zhu, and Radu Soricut. 2019. A Case Study on Combining ASR and Visual Features for Generating Instructional Video Captions. In Proceedings of the 23rd Conference on Computational Natural Language Learning (CoNLL). 419-429.

[16] Chiori Hori, Takaaki Hori, Teng-Yok Lee, Ziming Zhang, Bret Harsham, John R Hershey, Tim K Marks, and Kazuhiko Sumi. 2017. Attention-based multimodal fusion for video description. In Proceedings of the IEEE international conference on computer vision. 4193-4202.

[17] Sergey Ioffe and Christian Szegedy. 2015. Batch normalization: Accelerating deep network training by reducing internal covariate shift. arXiv preprint arXiv:1502.03167 (2015)

[18] Yunjae Jung, Donghyeon Cho, Sanghyun Woo, and In So Kweon. 2020. Globaland-Local Relative Position Embedding for Unsupervised Video Summarization. ECCV (2020).

[19] Atsushi Kanehira, Luc Van Gool, Yoshitaka Ushiku, and Tatsuya Harada. 2018. Viewpoint-Aware video summarization. In Proceedings of the IEEE Conference on Computer Vision and Pattern Recognition. 7435-7444.

[20] Evangelos Kazakos, Arsha Nagrani, Andrew Zisserman, and Dima Damen. 2019 Epic-fusion: Audio-visual temporal binding for egocentric action recognition. In Proceedings of the IEEE International Conference on Computer Vision. 5492-5501.

[21] Ranjay Krishna, Kenji Hata, Frederic Ren, Li Fei-Fei, and Juan Carlos Niebles. 2017. Dense-captioning events in videos. In ICCV. 706-715.

[22] Haoran Li, Junnan Zhu, Tianshang Liu, Jiajun Zhang, Chengqing Zong, et al. 2018. Multi-modal Sentence Summarization with Modality Attention and Image Filtering.. In IFCAI. 4152-4158.

[23] Haoran Li, Junnan Zhu, Cong Ma, Jiajun Zhang, and Chengqing Zong. 2017. Multi-modal summarization for asynchronous collection of text, image, audio and video. In Proceedings of the 2017 Conference on Empirical Methods in Natural Language Processing. 1092-1102.
[24] Chin-Yew Lin and Franz Josef Och. 2004. Automatic evaluation of machine translation quality using longest common subsequence and skip-bigram statistics. In Proceedings of the 42nd Annual Meeting of the Association for Computational Linguistics (ACL-04). 605-612.

[25] Nayu Liu, Xian Sun, Hongfeng Yu, Wenkai Zhang, and Guangluan Xu. 2020. Multistage Fusion with Forget Gate for Multimodal Summarization in OpenDomain Videos. In Proceedings of the 2020 Conference on Empirical Methods in Natural Language Processing (EMNLP). 1834-1845.

[26] Peter J Liu, Mohammad Saleh, Etienne Pot, Ben Goodrich, Ryan Sepassi, Lukasz Kaiser, and Noam Shazeer. 2018. Generating wikipedia by summarizing long sequences. ICLR (2018).

[27] Jiasen Lu, Dhruv Batra, Devi Parikh, and Stefan Lee. 2019. Vilbert: Pretraining task-agnostic visiolinguistic representations for vision-and-language tasks. In Advances in Neural Information Processing Systems. 13-23.

[28] Zheng Lu and Kristen Grauman. 2013. Story-driven summarization for egocentric video. In Proceedings of the IEEE Conference on Computer Vision and Pattern Recognition. 2714-2721.

[29] Shuming Ma, Lei Cui, Damai Dai, Furu Wei, and Xu Sun. 2019. Livebot: Generating live video comments based on visual and textual contexts. In Proceedings of the AAAI Conference on Artificial Intelligence, Vol. 33. 6810-6817.

[30] Antoine Miech, Jean-Baptiste Alayrac, Lucas Smaira, Ivan Laptev, Josef Sivic, and Andrew Zisserman. 2020. End-to-end learning of visual representations from uncurated instructional videos. In Proceedings of the IEEE/CVF Conference on Computer Vision and Pattern Recognition. 9879-9889.

[31] Antoine Miech, Dimitri Zhukov, Jean-Baptiste Alayrac, Makarand Tapaswi, Ivan Laptev, and Josef Sivic. 2019. Howto100m: Learning a text-video embedding by watching hundred million narrated video clips. In Proceedings of the IEEE international conference on computer vision. 2630-2640.

[32] Arthur G Money and Harry Agius. 2008. Video summarisation: A conceptual framework and survey of the state of the art. fournal of visual communication and image representation 19, 2 (2008), 121-143.

[33] N Moratanch and S Chitrakala. 2017. A survey on extractive text summarization. In 2017 international conference on computer, communication and signal processing (ICCCSP). IEEE, 1-6.

[34] Shruti Palaskar, Jindřich Libovickỳ, Spandana Gella, and Florian Metze. 2019. Multimodal Abstractive Summarization for How2 Videos. In Proceedings of the 57th Annual Meeting of the Association for Computational Linguistics. 6587-6596.

[35] Yingwei Pan, Ting Yao, Houqiang Li, and Tao Mei. 2017. Video captioning with transferred semantic attributes. In Proceedings of the IEEE conference on computer vision and pattern recognition. 6504-6512.

[36] Kishore Papineni, Salim Roukos, Todd Ward, and Wei-Jing Zhu. 2002. BLEU: a method for automatic evaluation of machine translation. In Proceedings of the 40th annual meeting of the Association for Computational Linguistics. 311-318.

[37] Ramakanth Pasunuru and Mohit Bansal. 2018. Game-Based Video-Context Dialogue. In Proceedings of the 2018 Conference on Empirical Methods in Natural Language Processing. 125-136.

[38] Vasili Ramanishka, Abir Das, Dong Huk Park, Subhashini Venugopalan, Lisa Anne Hendricks, Marcus Rohrbach, and Kate Saenko. 2016. Multimodal video description. In Proceedings of the 24th ACM international conference on Multimedia. 1092-1096.

[39] Anirudh Ravula, Chris Alberti, Joshua Ainslie, Li Yang, Philip Minh Pham, Qifan Wang, Santiago Ontanon, Sumit Kumar Sanghai, Vaclav Cvicek, and Zach Fisher. 2020. ETC: Encoding Long and Structured Inputs in Transformers. EMNLP (2020).

[40] Ramon Sanabria, Ozan Caglayan, Shruti Palaskar, Desmond Elliott, Loïc Barrault, Lucia Specia, and Florian Metze. 2018. How2: A Large-scale Dataset for Multimodal Language Understanding. In NeurIPS.

[41] Peter Shaw, Jakob Uszkoreit, and Ashish Vaswani. 2018. Self-Attention with Relative Position Representations. In Proceedings of the 2018 Conference of the North American Chapter of the Association for Computational Linguistics: Human Language Technologies, Volume 2 (Short Papers). 464-468.

[42] Zhiqiang Shen, Jianguo Li, Zhou Su, Minjun Li, Yurong Chen, Yu-Gang Jiang, and Xiangyang Xue. 2017. Weakly supervised dense video captioning. In Proceedings of the IEEE Conference on Computer Vision and Pattern Recognition. 1916-1924.

[43] Rakshith Shetty, Marcus Rohrbach, Lisa Anne Hendricks, Mario Fritz, and Bernt Schiele. 2017. Speaking the same language: Matching machine to human captions by adversarial training. In Proceedings of the IEEE International Conference on Computer Vision. 4135-4144.

[44] Botian Shi, Lei Ji, Yaobo Liang, Nan Duan, Peng Chen, Zhendong Niu, and Ming Zhou. 2019. Dense procedure captioning in narrated instructional videos. In Proceedings of the 57th Annual Meeting of the Association for Computational Linguistics. 6382-6391.

[45] Matthias Sperber, Jan Niehues, Graham Neubig, Sebastian Stüker, and Alex Waibel. 2018. Self-Attentional Acoustic Models. Proc. Interspeech 2018 (2018), 3723-3727.

[46] Weijie Su, Xizhou Zhu, Yue Cao, Bin Li, Lewei Lu, Furu Wei, and Jifeng Dai. 2019. VL-BERT: Pre-training of Generic Visual-Linguistic Representations. In International Conference on Learning Representations. 
[47] Chen Sun, Austin Myers, Carl Vondrick, Kevin Murphy, and Cordelia Schmid. 2019. Videobert: A joint model for video and language representation learning. In Proceedings of the IEEE International Conference on Computer Vision. 7464-7473.

[48] Yapeng Tian, Jing Shi, Bochen Li, Zhiyao Duan, and Chenliang Xu. 2018. Audiovisual event localization in unconstrained videos. In Proceedings of the European Conference on Computer Vision (ECCV). 247-263.

[49] Ashish Vaswani, Noam Shazeer, Niki Parmar, Jakob Uszkoreit, Llion Jones, Aidan N Gomez, Łukasz Kaiser, and Illia Polosukhin. 2017. Attention is all you need. In Advances in neural information processing systems. 5998-6008.

[50] Ramakrishna Vedantam, C Lawrence Zitnick, and Devi Parikh. 2015. Cider Consensus-based image description evaluation. In Proceedings of the IEEE conference on computer vision and pattern recognition. 4566-4575.

[51] Subhashini Venugopalan, Marcus Rohrbach, Jeffrey Donahue, Raymond Mooney Trevor Darrell, and Kate Saenko. 2015. Sequence to sequence-video to text. In Proceedings of the IEEE international conference on computer vision. 4534-4542.

[52] William Yang Wang, Yashar Mehdad, Dragomir Radev, and Amanda Stent. 2016 A low-rank approximation approach to learning joint embeddings of news stories and images for timeline summarization. In Proceedings of the 2016 Conference of the North American Chapter of the Association for Computational Linguistics: Human Language Technologies. 58-68.

[53] Yu Wu, Linchao Zhu, Yan Yan, and Yi Yang. 2019. Dual attention matching for audio-visual event localization. In Proceedings of the IEEE International Conference on Computer Vision. 6292-6300.

[54] Jun Xu, Tao Mei, Ting Yao, and Yong Rui. 2016. Msr-vtt: A large video description dataset for bridging video and language. In Proceedings of the IEEE conference on computer vision and pattern recognition. 5288-5296.

[55] Jun Xu, Ting Yao, Yongdong Zhang, and Tao Mei. 2017. Learning multimodal attention LSTM networks for video captioning. In Proceedings of the 25th ACM international conference on Multimedia. 537-545.

[56] Baosong Yang, Zhaopeng Tu, Derek F Wong, Fandong Meng, Lidia S Chao, and Tong Zhang. 2018. Modeling localness for self-attention networks. arXiv preprint
arXiv:1810.10182 (2018)

[57] Li Yao, Atousa Torabi, Kyunghyun Cho, Nicolas Ballas, Christopher Pal, Hugo Larochelle, and Aaron Courville. 2015. Describing videos by exploiting temporal structure. In Proceedings of the IEEE international conference on computer vision. 4507-4515.

[58] Shoou-I Yu, Lu Jiang, and Alexander Hauptmann. 2014. Instructional videos for unsupervised harvesting and learning of action examples. In Proceedings of the 22nd ACM international conference on Multimedia. 825-828.

[59] Manzil Zaheer, Guru Guruganesh, Avinava Dubey, Joshua Ainslie, Chris Alberti, Santiago Ontanon, Philip Pham, Anirudh Ravula, Qifan Wang, Li Yang, et al. 2020. Big bird: Transformers for longer sequences. arXiv preprint arXiv:2007.14062 (2020).

[60] Alireza Zareian, Haoxuan You, Zhecan Wang, and Shih-Fu Chang. 2020. Learning Visual Commonsense for Robust Scene Graph Generation. ECCV (2020).

[61] Shengyu Zhang, Tan Jiang, Tan Wang, Kun Kuang, Zhou Zhao, Jianke Zhu, Jin Yu, Hongxia Yang, and Fei Wu. 2020. Devlbert: Learning deconfounded visiolinguistic representations. In Proceedings of the 28th ACM International Conference on Multimedia. 4373-4382.

[62] Luowei Zhou, Hamid Palangi, Lei Zhang, Houdong Hu, Jason J Corso, and Jianfeng Gao. 2020. Unified Vision-Language Pre-Training for Image Captioning and VQA.. In AAAI. 13041-13049.

[63] Luowei Zhou, Chenliang $\mathrm{Xu}$, and Jason J Corso. 2018. Towards Automatic Learning of Procedures from Web Instructional Videos. In AAAI Conference on Artificial Intelligence.

[64] Luowei Zhou, Yingbo Zhou, Jason J Corso, Richard Socher, and Caiming Xiong. 2018. End-to-end dense video captioning with masked transformer. In Proceedings of the IEEE Conference on Computer Vision and Pattern Recognition. 8739-8748.

[65] Junnan Zhu, Yu Zhou, Jiajun Zhang, Haoran Li, Chengqing Zong, and Changliang Li. 2020. Multimodal summarization with guidance of multimodal reference. In Proceedings of the AAAI Conference on Artificial Intelligence, Vol. 34. 9749-9756. 